\title{
Effects of Propeller Slipstream of Distributed Electric Propulsion on the Wing-Flap System
}

Semyon Mikhalyov ${ }^{1}$, Andrey Dunaevsky, Leonid Teperin, Roman Vasilyev, and Andrey Redkin

TsAGI, 1, Zhukovsky str, Zhukovsky town, Moscow region, Russia

\begin{abstract}
The studies are aimed at investigating the mechanism to ensure the high lift by installing propellers in front of the wing and using interference effects. The model of the wing section of a regional STOL aircraft with DEPS is considered. The wing section is equipped with a trailing edge device (Fowler flap) and DEPS consisting of motors with propellers. Parametric and experimental studies of the interaction between DEPS propellers and the regional STOL aircraft wing-flap system were carried out. The development and verification of aerodynamic calculation method for the wing section at low Reynolds numbers were performed. The comparison with the wind tunnel test data obtained at TSAGI was made. It was found that: - for fixed power of propellers there is an optimal propeller diameter ensuring maximum increase in the wing section lift; - at low thrust the total lift weakly depends on DEPS inclination angle.
\end{abstract}

\section{Description of design model}

The model of the wing section of a regional STOL aircraft with DEPS is considered. The aircraft is a monoplane of normal configuration with upper unswept wing; cruising engines are also installed on the wing. The $\mathrm{T}$-shaped tail consists of a vertical tail with a rudder and a horizontal tail with an elevator at the end of the fin. For regional turboprop aircraft, it is of interest to consider the transition from a twin-propeller configuration to aircraft with DEPS. For this purpose, calculation and experimental studies of the wing section with a flap and propellers installed in front of the leading edge were carried out.

P4-14M airfoil section developed at TsAGI was selected as the main one [1]. A singleslot retractable Fowler flap with a $30 \%$ chord is used as a high-lift wing device (Fig. 1).

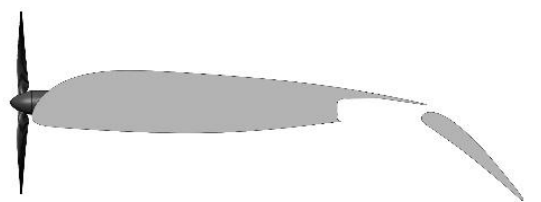

a) take-off and landing configuration (with flap and propeller)

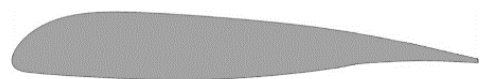

b) cruise configuration

Fig. 1. Regional STOL aircraft wing configuration

Practical realization of the jet flows simulation is performed by numerical solution of problems using averaged Reynolds number calculation method for turbulent flows - RANS (ANSYS CFX, TSAGI license No. 501024). The turbulence model used is the Menter shear

${ }^{1}$ Corresponding author: semyon.mikhalyov@tsagi.ru 
stress transport SST $k-\omega$ [2]. This method is the most suitable due to the relative quickness of solution and little computational effort. The solution is time invariant, with the transition from a continuous task in time and space to its discrete analogue.

One of the most important issues, along with the turbulence model choice is the choice of mesh parameters. These and many other questions can be answered positively only if the calculation method for jet flows simulation is successfully verified. The verification was carried out using test results obtained at TSAGI (Russia, Zhukovsky) in 2018.

\section{Description of TsAGI experiment}

A photograph of the wing section model with the system of blowing by the propellers slipstream installed in the wind tunnel with semi-axes dimensions of the test section $2 \times 1.16$ $\mathrm{m}$ is shown in Fig. 2.

The wing has a rectangular plan form, a chord of $0.72 \mathrm{~m}$, a wingspan of $1.2 \mathrm{~m}$ and is bounded on both sides by cutting disks with a diameter of $1 \mathrm{~m}$ and a thickness of $15 \mathrm{~mm}$. The wing is equipped with the Fowler flap and DEPS consisting of four electric motors located inside the wing torsion box (Fig. 3). The power plant consists of four two-bladed co- rotating propellers with a diameter of $0.3 \mathrm{~m}$, and driven by electric motors. During the tests, the engine inclination angle $i=-10^{\circ}$ and $-30^{\circ}$ and the flap angle $\delta_{\text {flap }}=0^{\circ}, 15^{\circ}, 35^{\circ}$ and $40^{\circ}$ relative to the wing chord were varied. The flow velocity ranged from 10 to $40 \mathrm{~m} / \mathrm{s}$, the angles of attack ranged from $-10^{\circ}$ to $+24^{\circ}$.

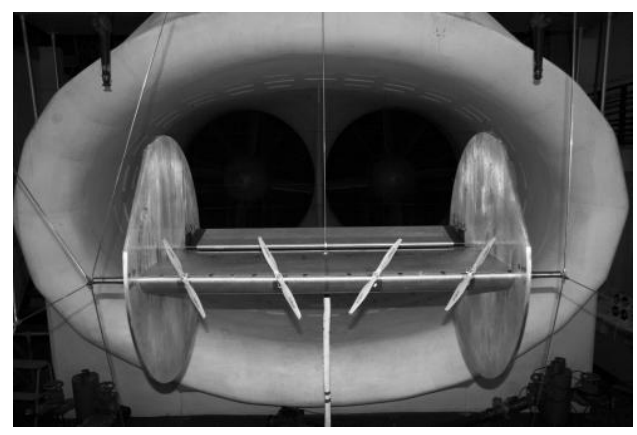

Fig. 2. Model of the wing section with DEPS in the wind tunnel (inverted)
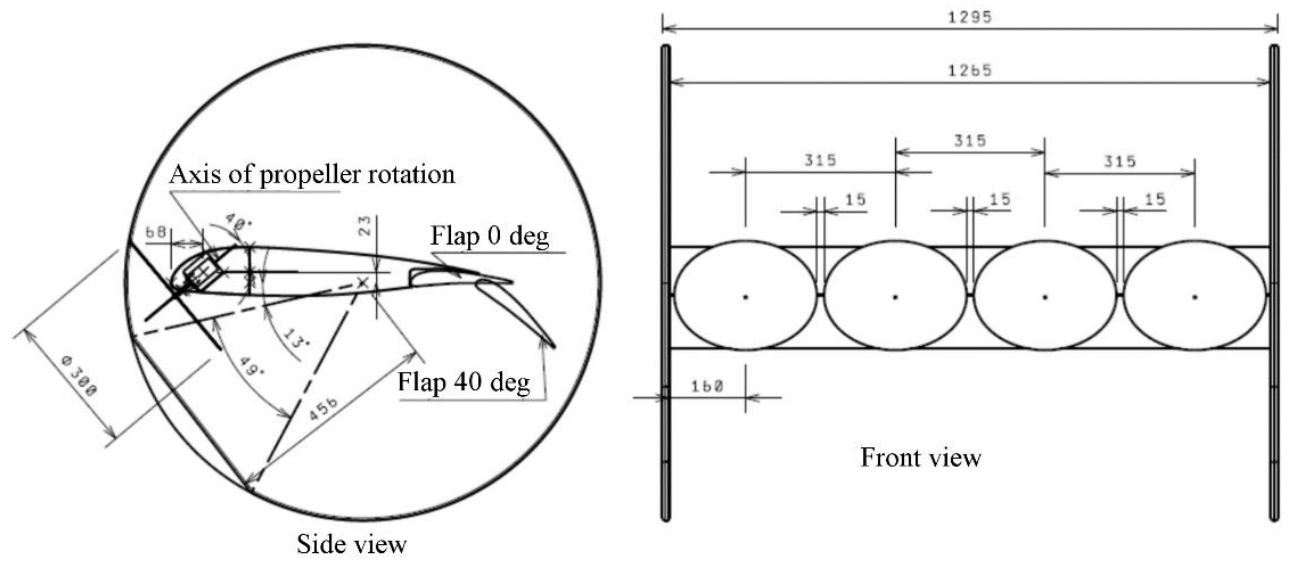

Fig. 3. General drawing and overall dimensions of the wing section model with DEPS 


\section{Simulation of ideal propeller operation}

The specific feature of the engine simulation is that, unlike an airframe, on the surface of which the boundary condition of non-permeability or flow attachment is fulfilled, the boundary conditions on the engine elements are not so obvious and require individual consideration. To simplify the further statement of the problem, the engine is divided into a power unit and a thrust unit for clarity.

We will not consider the engine unit that generates power, and will represent the engine thrust unit in the form of a coaxial propeller and propeller with constant pressure drop across the swept surface. Let us consider the active disk method. In references [3, 4], a smallthickness disk located in the plane of the propeller is introduced into the solution region. On the front and rear side of the disk, the boundary condition of the given velocity and flow rate is set. We will demonstrate the disadvantages of propeller simulation with active flat-faceted disk using a flat active section in incompressible 2D flow, in which the jet velocity at infinity is twice higher than the incident flow velocity. Fig. 4 shows the distribution of relative velocity along the line passing through the center. The solid line indicates the theoretical distribution:

$$
\begin{aligned}
& x<0, V_{x}=V_{\infty}+\frac{\left(V_{\infty}-V_{j e t}\right)}{\pi} \operatorname{arctg} \frac{D}{2 x}, \\
& x>0, V_{x}=V_{j e t}+\frac{\left(V_{\infty}-V_{j e t}\right)}{\pi} \operatorname{arctg} \frac{D}{2 x} .
\end{aligned}
$$

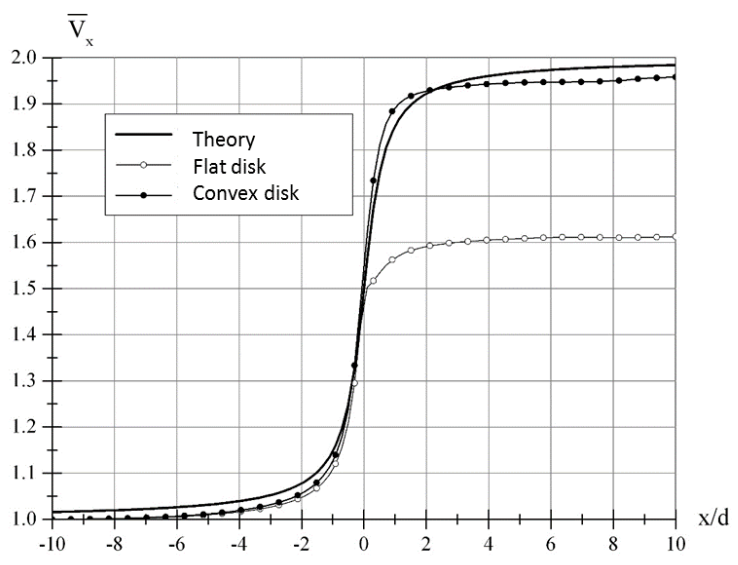

Fig. 4. Relative velocity distribution along a horizontal line passing through the active section centre

The empty circles show the distribution obtained from the ANSYS CFX calculation based on the flat active disk simulation. The incident flow velocity was $10 \mathrm{~m} / \mathrm{s}$ and the jet velocity at infinity was $20 \mathrm{~m} / \mathrm{s}$. The velocity in front of the disc is in good agreement with the theoretical distribution, and the velocity behind the disc is much less.

In Fig. 4, the black circles show the velocity distribution obtained by simulating the propeller not by a plane but convex towards the incident flow active section. This distribution was obtained as follows. Two domains were introduced in the ANSYS CFX program: the main domain was in the form of $2 \mathrm{D}$ rectangular and the additional domain (Fig. 5) was in the form of a lens with a convex side to the inflow and with a flat opposite side. On the outer convex side, the boundary condition of the outflow from the main domain with the normal velocity $V_{\mathrm{n}}=\left(V_{\infty}+V_{\text {jet }}\right) / 2$ was set. On the inner convex side, the boundary condition of the inflow with the same normal velocity $V_{\mathrm{n}}$ was set. On the flat side of the lens, the liquid-liquid interface was set on both sides. To construct the shape of the convex part of the lens, we 
determine the velocities induced by the distribution of sinks of constant intensity $\sigma$ on the active section surface out of the jet $(x=-0)$ :

$$
V_{y}=\frac{\partial \varphi_{\sigma}}{\partial y}=-\frac{\sigma}{4 \pi} \ln \left[\frac{(y-D)^{2}+x^{2}}{y^{2}+x^{2}}\right], V_{x}=\frac{\partial \varphi_{\sigma}}{\partial x}=\frac{\sigma}{2 \pi}\left[\operatorname{arctg} \frac{y}{x}-\operatorname{arctg} \frac{y-D}{x}\right] .
$$

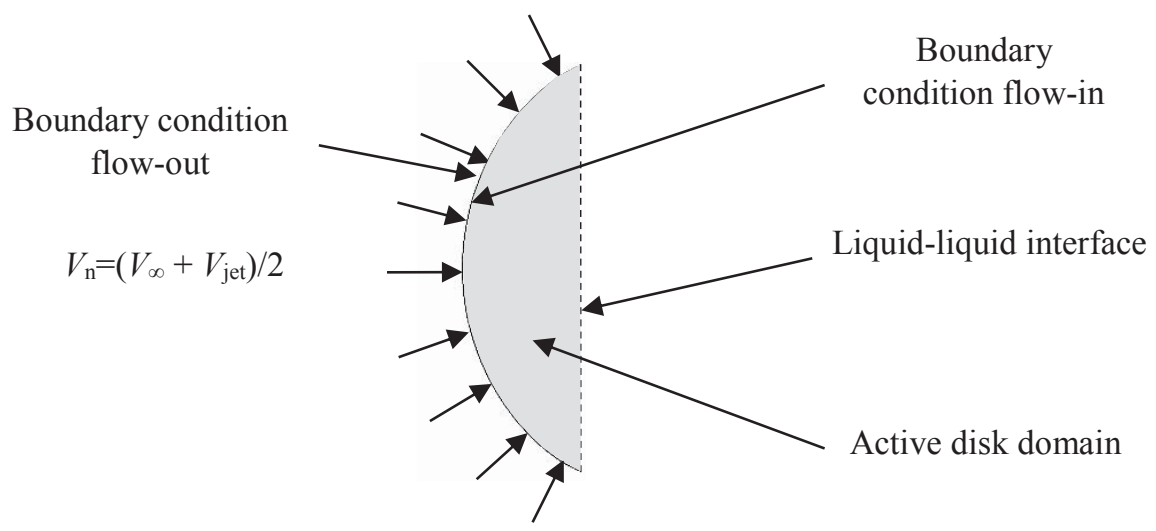

Fig. 5. Active section in the form of lens

The total velocities on the active section from the side of the inflow $(x=-0)$ filled with sinks will be equal to:

$$
V_{y}=-\frac{\left(V_{\infty}-V_{j e t}\right)}{4 \pi} \ln \left[\frac{(y-D)^{2}}{y^{2}}\right], V_{x}=\frac{\left(V_{\infty}+V_{j e t}\right)}{2} .
$$

Integrating the equation

$$
\frac{d x}{d y}=-\frac{\left(V_{\infty}-V_{j e t}\right)}{2 \pi\left(V_{\infty}+V_{j e t}\right)} \ln \left[\frac{(y-D)^{2}}{y^{2}}\right],
$$

we obtain a curve orthogonal to the streamlines

$$
x(y)=\frac{\left(V_{\infty}-V_{j e t}\right)}{\pi\left(V_{\infty}+V_{j e t}\right)}\left[(D-y) \ln \left(1-\frac{y}{D}\right)+y \ln \frac{y}{D}\right],
$$

which is used for constructing additional domain that simulates the propeller operation.

Let us extend the obtained result for $3 \mathrm{D}$ active disk. It is rather difficult to derive exact formulas for the velocities induced by the circular disk of sinks. Only the analytical expression for the relative axial velocity along the line passing through the center of the disk is known [5]:

$$
\bar{V}_{x}=0.5\left(\bar{V}_{j e t}+1\right)+\frac{0.5\left(\bar{V}_{j e t}-1\right) \bar{x}}{\sqrt{\bar{x}^{2}+1}},
$$

where $\bar{x}$ is a distance to the active disk referred to its radius.

To simplify the task, we replace the circular active disk with a polygon, which can be represented as a set of triangles (Fig. 6). The potential and components of the velocities induced by the angle uniformly filled with sources or sinks are (Fig. 6):

$$
\begin{gathered}
\varphi_{i}(x, y, z)=-\frac{\sigma}{4 \pi} \iint_{S} \frac{d \xi d \eta}{\sqrt{(x-\xi)^{2}+(y-\eta)^{2}+z^{2}}}, \\
u_{i}=\frac{\partial \varphi_{i}}{\partial x}, v_{i}=\frac{\partial \varphi_{i}}{\partial y}, w_{i}=\frac{\partial \varphi_{i}}{\partial z},
\end{gathered}
$$




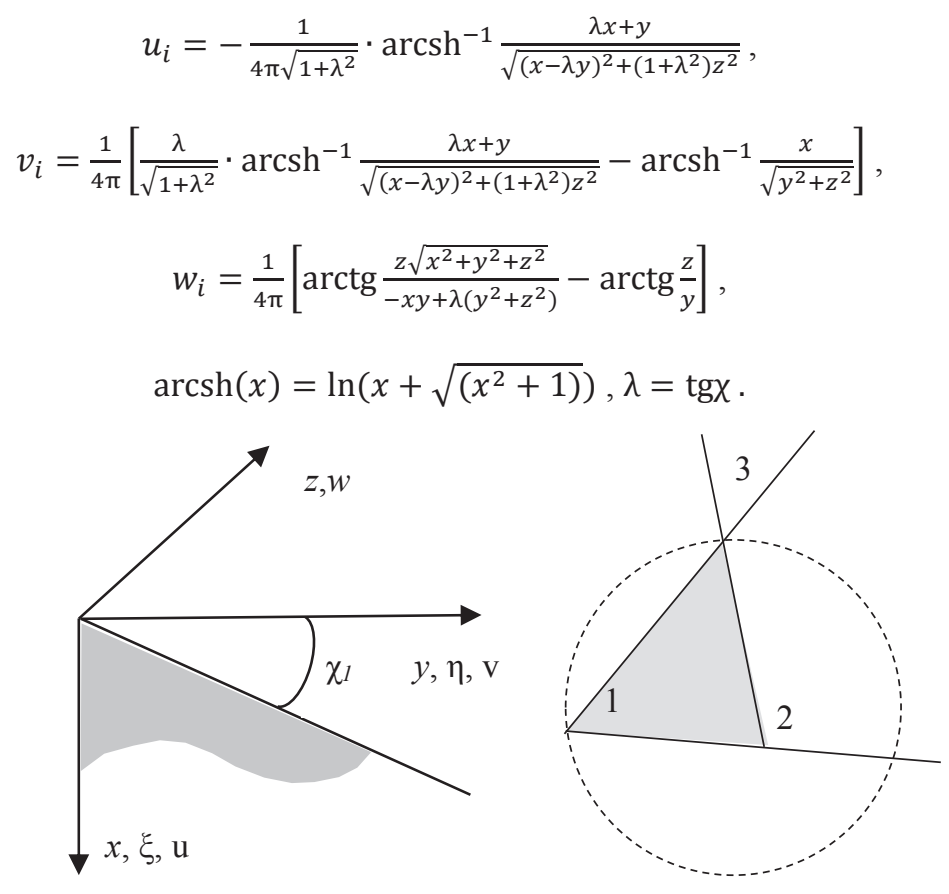

Fig. 6. Angle filled with sources

The velocities induced by one triangle can be defined as a combination of three angles:

$$
u=u_{1}-u_{2}+u_{3}, v=v_{1}-v_{2}+v_{3}, w=w_{1}-w_{2}+w_{3} .
$$

Replacing a circular active disk with a large number of triangles makes it possible to obtain velocities induced by a circular active disk with the required accuracy. Integrating the equation

$$
\frac{d x}{d y}=\frac{\left(1-\bar{V}_{j e t}\right) v}{1+\left(1-\bar{V}_{j e t}\right) w}
$$

along the vertical line passing through the disk center we obtain a section of the convex part of the lens to build an additional domain.

\section{Verification of calculation method}

In this subsection the results of numerical simulation studies of jet flows and their interference with wing-flap section (with single-slot flap deflected by 0 and $40^{\circ}$ and propeller inclination angle by $-30^{\circ}$ ) are given. The comparison is made with the wind tunnel experiment. The calculation results are presented in Figs. $8-10$. There is a satisfactory agreement with the lift coefficient calculation. Therefore, the root-mean-square error is satisfactory and falls within $\sim 5 \%$. It proves the correctness of the chosen model for the active disk, the turbulence model, and steps taken for meshing. Fig. 7 shows the lift coefficient $c_{\mathrm{L}}$ of the wing section without blowing; Figs. 8 and 9 - with blowing at propeller specific thrust $B=0.5$ (thrust, related to dynamic pressure and propeller-swept area). 


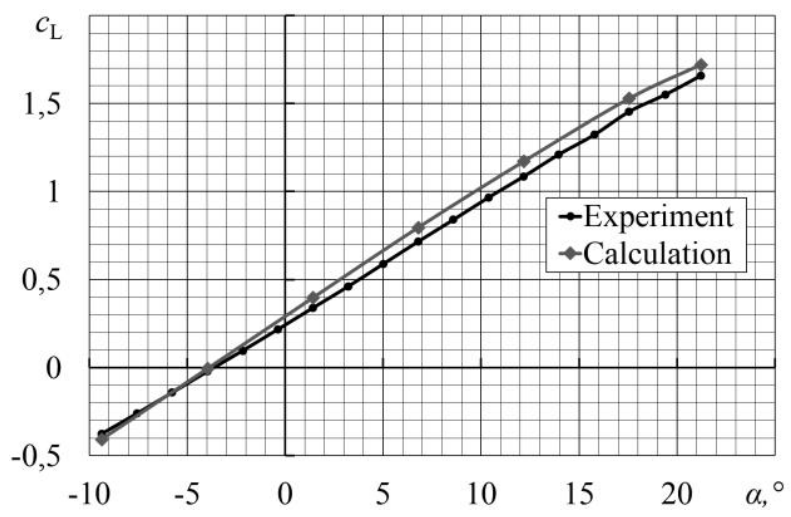

Fig. 7. Wing section lift coefficient $c_{\mathrm{L}}$ vs angle of attack $\alpha$ without blowing

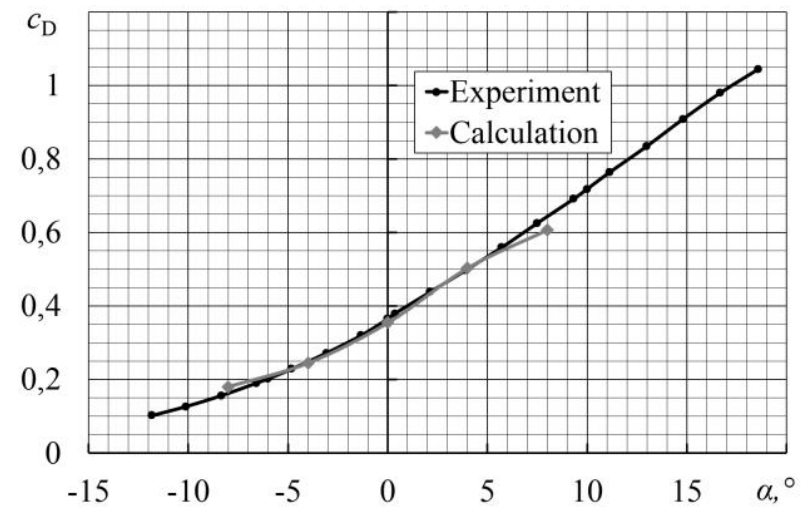

Fig. 8. Wing section drag coefficient $c_{\mathrm{D}}$ vs angle of attack $\alpha$ at specific propeller thrust $B=0.5$

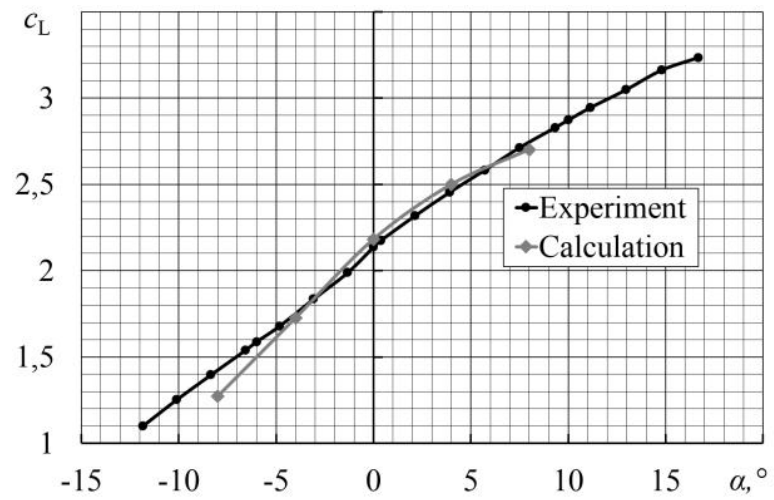

Fig. 9. Wing section lift coefficient $c_{\mathrm{L}}$ vs angle of attack $\alpha$ at specific propeller thrust $B=0.5$

As it is shown in a number of experimental works, an important parameter for configurations with blowing is the jet angular orientation under take-off and landing conditions [6]. Fig. 10 shows the experimental dependence of the lift coefficient $c_{\mathrm{L}}$ of the wing section at specific propeller thrust $B=1.15$. Reducing the inclination angle of the electric motor axis from -10 to -30 degrees practically does not lead to a change in lift. Thus, the total lift weakly depends on the engine axis inclination angle relative to the wing at low specific thrust. 


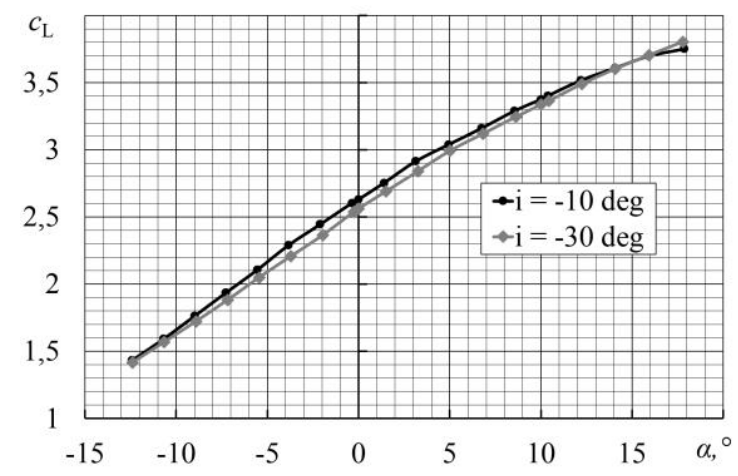

Fig. 10. Experimental dependence of wing section lift coefficient $c_{\mathrm{L}}$ on angle of attack $\alpha$ at specific propeller thrust $B=1.15$

\section{Parametric studies}

Thus, having an adequate model of a propeller and FEM parameters of the wing section obtained by comparing with the experiment data, further calculation studies can be performed with satisfactory accuracy.

The studies were carried out to select the propeller diameter $D$ (which is associated with the number of propellers $\mathrm{n}$ and the specific thrust $B$ ) to maximize the lift coefficient $c_{\mathrm{L}}$ of the streamlined airfoil, taking into account the propeller thrust.

The values of $c_{\mathrm{L}}$ coefficient are not identical for different diameters for the same input power $N$. For fixed power of propellers there is an optimal diameter $D$ to ensure maximum increase in the wing section lift. This is explained by the fact that the fixation of the excess jet momentum (specific thrust $B$ ) imposes a relationship on the relative diameter of the active disk $\bar{D}=D / b$ and the relative velocity in the jet $\bar{V}_{j}=V_{j} / V_{0}[7]$ :

$$
B=2 \bar{D}_{j}\left(\bar{V}_{j}-1\right) \text {. }
$$

Therefore, at $\bar{D} \rightarrow \infty V_{j} \rightarrow V_{0}$ there is no increase in lift. Reducing the active disk diameter, we come to a certain value $\bar{D}=\bar{D}^{*}$ when the increase in lift is maximum. With a further decrease in diameter, the influence of the jet boundaries becomes apparent, and the lift increment decreases. CFD studies show that the optimal diameter is $\bar{D}^{*} \approx 0.5$ at $B \sim 3$ (Fig. 11).

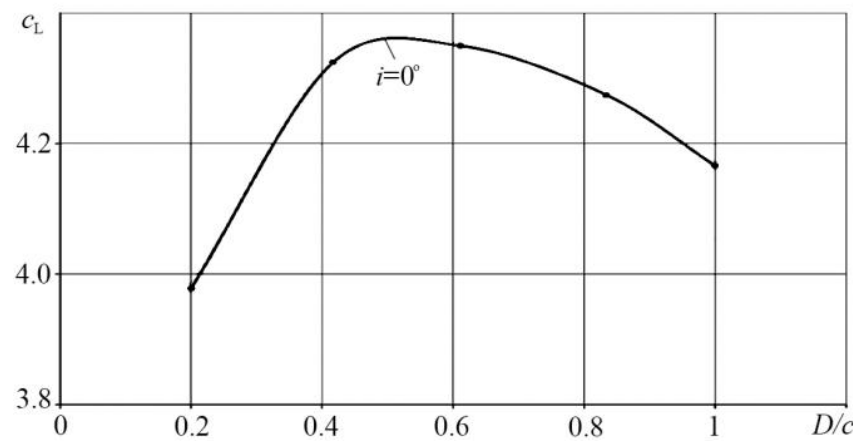

Fig. 11. Dependence of the lift coefficient of a wing section with a single-slot flap on nondimensional propeller diameter at angle of attack $=0^{\circ}$. Flap deflection is $40^{\circ}$. Total input power is constant and equal to $N=5.4 \mathrm{~kW}$ 


\section{Conclusion}

The domain configuration of a special form was developed, which makes it possible to obtain a velocity field in 2D and 3D flows close to theoretical Froude model of an ideal propeller for solving problems based on Navier-Stokes equations in the ANSYS CFX calculation system.

For an adequate application of the active disk model in computational fluid dynamics problems, using finite-difference methods, the technique was developed where the domain is represented as a "lens" on which the boundary conditions of a given velocity or flow rate are fulfilled. The "lens" shape depends on the propeller diameter, inflow velocity and jet velocity at infinity.

The dependence of the wing lift increase on non-dimensional propeller diameter (related to the wing chord) was determined for a fixed input power. It was shown that for specific thrust (related to dynamic pressure and active disk area) $B=3$, the optimal value of nondimensional propeller diameter is 0.5 .

It was found that with low thrust the total lift weakly depends on the DEPS inclination angle.

\section{References}

1. Yu.S. Mikhaylov, Aerodynamic design of high-lift airfoil sections for general aviation aircraft, Air Fleet Engineering, 1 (1999)

2. F. Menter, Two-Equation Eddy-Viscosity Turbulence Models for Engineering Applications, AIAA Journal, 32 (8), 1598-1605, (1994)

3. A.A. Razov, Numerical analysis of propeller positioning efficiency in a viscous wake using the Navier-Stokes equations. TsAGI Uchenye Zapiski, XL, 3, 28-35 (2009)

4. S.G. Ignatyev, L.N. Teperina, M. El-Salamoni, On operation of the propulsor behind the trailing edge of the airfoil section at transonic speed. Uchenye Zapiski TsAGI, 3, 310 (2017)

5. D. Kyukheman, I. Veber, Aerodynamics of aircraft engines, IL, Moscow, 388 (1956)

6. C.L. Jr. Gentry, M.A. Takallu, Z.T. Applin, Aerodynamic Characteristics of a PropellerPowered High-Lift Semispan Wing, NASA Technical Memorandum, 68 (1994)

7. V.I. Babkin, S.M. Belotserkovsky, V.V. Gulyaev, A.V. Dvorak, Jets and lifting surfaces: Computer simulation, Moscow, Nauka, 208 (1989) 